\title{
Robust Fuzzy Control for Fractional-Order Uncertain Hydroturbine Regulating System with Random Disturbances
}

\author{
Fengjiao Wu, ${ }^{1}$ Guitao Zhang, ${ }^{2}$ and Zhengzhong Wang ${ }^{1}$ \\ ${ }^{1}$ Department of Electrical Engineering, Northwest A\&F University, Shaanxi, Yangling 712100, China \\ ${ }^{2}$ School of Electrical Engineering, Xian Jiaotong University, Shaanxi, Xian 710049, China \\ Correspondence should be addressed to Zhengzhong Wang; wangzz0910@163.com
}

Received 4 May 2016; Accepted 8 September 2016

Academic Editor: Fazal M. Mahomed

Copyright (c) 2016 Fengjiao Wu et al. This is an open access article distributed under the Creative Commons Attribution License, which permits unrestricted use, distribution, and reproduction in any medium, provided the original work is properly cited.

\begin{abstract}
The robust fuzzy control for fractional-order hydroturbine regulating system is studied in this paper. First, the more practical fractional-order hydroturbine regulating system with uncertain parameters and random disturbances is presented. Then, on the basis of interval matrix theory and fractional-order stability theorem, a fuzzy control method is proposed for fractional-order hydroturbine regulating system, and the stability condition is expressed as a group of linear matrix inequalities. Furthermore, the proposed method has good robustness which can process external random disturbances and uncertain parameters. Finally, the validity and superiority are proved by the numerical simulations.
\end{abstract}

\section{Introduction}

Due to the advantages of describing actual projects, especially for the description of memory and hereditary properties of numerous materials and processes $[1,2]$, fractional calculus has attracted more and more people's attention. It has been verified that many practical systems could be elegantly expressed with fractional calculus, such as power system [3], permanent magnet synchronous motor system [4], mechanical system [5], and chemical processing system [6].

The hydroturbine regulating system is a core component for safe and stable operation of hydropower station system. For a long time up to now, the integer-order model is usually adopted [7-9]. However, as we all know, the hydroturbine regulating system is integration of hydraulic, mechanical, and electrical parts. This complex composition makes it a strong coupling, nonlinear, and nonminimum phase system [10-12]. So the integer-order model may not be suitable for perfectly describing the hydroturbine regulating system. Besides, due to the memory character and history-dependence of hydraulic servo system, the more practical fractional-order model is considered accordingly in this study. Many studies have shown that the hydroturbine regulating system may exhibit irregular nonlinear vibrations when the system is under parameter variations and external random disturbances [13-15]. Therefore, it is very important to design nonlinear controller for the stable operation of fractional-order hydroturbine regulating system. Until now, some nonlinear control schemes have been proposed for fractional-order systems, such as sliding mode control [16], predictive control [17], adaptive control [18], and pinning control [19]. However, few of the above-mentioned methods consider the uncertainty and random disturbances.

Fuzzy control is a robust method, which can deal with external disturbances [20-22]. Besides, with the help of fuzzy linearization and linear matrix theory, the uncertain parameters can be well processed [23-25]. There have been many results applying fuzzy control to integer-order nonlinear systems [26-29]. However, there is little literature about fractional-order nonlinear fuzzy control and there is almost no relevant result for fractional-order hydroturbine regulating system.

According to the above analysis, some advantages are shown in this study. Firstly, the fractional-order hydroturbine regulating system with uncertain parameters and random 
disturbances is presented, which is more in accordance with practical engineering. Secondly, a fuzzy control method is proposed for fractional-order hydroturbine regulating system, and the stability condition is expressed as a group of linear matrix inequalities (LMIs). Furthermore, the proposed method has good robustness which can process external random disturbances and uncertain parameters. Even if the system is with six uncertain parameters, the controller designed for fractional-order hydroturbine regulating system is still valid. Lastly, numerical simulations have demonstrated the effectiveness and superiority when compared with traditional PID control method.

The rest of this paper is organized as follows. In Section 2, the fractional-order hydroturbine regulating system is introduced. Section 3 presents the robust fuzzy controller design for fractional-order hydroturbine regulating system. Simulations are shown in Section 4. And the paper is ended in Section 5.

\section{The Fractional-Order Hydroturbine Regulating System}

The integer-order hydroturbine regulation system is given as [30]

$$
\begin{aligned}
\frac{d \delta}{d t} & =\omega_{0} \omega \\
\frac{d \omega}{d t} & =\frac{1}{T_{a b}}\left(m_{t}-D \omega-\frac{E_{q}^{\prime} V_{s}}{x_{d \Sigma}^{\prime}} \sin \delta\right. \\
& \left.-\frac{V_{s}^{2}}{2} \frac{x_{d \Sigma}^{\prime}-x_{q \Sigma}}{x_{d \Sigma}^{\prime} x_{q \Sigma}} \sin 2 \delta\right), \\
\frac{d m_{t}}{d t} & =\frac{1}{e_{q h} T_{w}}\left(-m_{t}+e_{y} y+\frac{e e_{y} T_{w}}{T_{y}} y\right), \\
\frac{d y}{d t} & =-\frac{1}{T_{y}} y
\end{aligned}
$$

where $\delta, \omega, m_{t}$, and $y$ are the rotor angle deviation of the generator, the relative deviation of the rotational speed of the generator, the hydroturbine output incremental torque deviation, and the incremental deviation of the guide vane opening, respectively.

The hydraulic servo system has significant historical reliance. Since it is a powerful advantage for fractional calculus to describe the function which has significant historical reliance, the fractional-order hydraulic servo system is adopted.

According to fractional calculus, the fractional-order hydraulic servo system is obtained as

$$
D^{\alpha} y=\frac{1}{T_{y}}(u-y),
$$

where $T_{y}$ is the major relay connector response time.
According to (1) and (2) and for convenience, we use $x_{1}, x_{2}, x_{3}, x_{4}$ to replace $\delta, \omega, m_{t}, y$. Then, the fractional-order mathematical model of hydroturbine regulation system could be represented as

$$
\begin{aligned}
\frac{d x_{1}}{d t} & =\omega_{0} x_{2}, \\
\frac{d x_{2}}{d t} & =\frac{1}{T_{a b}}\left(x_{3}-D x_{2}-\frac{E_{q}^{\prime} V_{s}}{x_{d \Sigma}^{\prime}} \sin x_{1}\right. \\
\left.-\frac{V_{s}^{2}}{2} \frac{x_{d \Sigma}^{\prime}-x_{q \Sigma}}{x_{d \Sigma}^{\prime} x_{q \Sigma}} \sin 2 x_{1}\right), & \frac{1}{d x_{3}} \frac{1}{d t}\left(-x_{3}+e_{y} x_{4}+\frac{e e_{y} T_{w}}{T_{y}} x_{4}\right), \\
\frac{d^{\alpha} x_{4}}{d t^{\alpha}} & =-\frac{1}{T_{y}} x_{4},
\end{aligned}
$$

where $\omega_{0}=314, T_{a b}=9.0 \mathrm{~s}, D=2.0, E_{q}^{\prime}=1.35, x_{d \Sigma}^{\prime}=1.15$, $x_{q \Sigma}=1.474, T_{w}=0.8 \mathrm{~s}, T_{y}=0.1 \mathrm{~s}, V_{s}=1.0, e_{q h}=0.5$, $e_{y}=1.0, e=0.7$, and $\alpha=0.9$.

Considering the universality of disturbances, the fractional-order hydroturbine regulating system could be represented as

$$
\begin{aligned}
\frac{d x_{1}}{d t} & =\omega_{0} x_{2}+\operatorname{rand}(1) \times x_{1}, \\
\frac{d x_{2}}{d t} & =\frac{1}{T_{a b}}\left(x_{3}-D x_{2}-\frac{E_{q}^{\prime} V_{s}}{x_{d \Sigma}^{\prime}} \sin x_{1}\right. \\
& \left.-\frac{V_{s}^{2}}{2} \frac{x_{d \Sigma}^{\prime}-x_{q \Sigma}}{x_{d \Sigma}^{\prime} x_{q \Sigma}} \sin 2 x_{1}\right)+\operatorname{rand}(1) \times x_{2}, \\
\frac{d x_{3}}{d t} & =\frac{1}{e_{q h} T_{w}}\left(-x_{3}+e_{y} x_{4}+\frac{e e_{y} T_{w}}{T_{y}} x_{4}\right)+\operatorname{rand}(1) \\
\quad \times x_{3} & \\
\frac{d^{\alpha} x_{4}}{d t^{\alpha}} & =-\frac{1}{T_{y}} x_{4}+\operatorname{rand}(1) \times x_{4} .
\end{aligned}
$$

Figure 1 shows the state trajectories of fractionalorder hydroturbine regulating system (4) with initial value $\left[\begin{array}{llll}x_{1} & x_{2} & x_{3} & x_{4}\end{array}\right]^{T}=\left[\begin{array}{llll}0.01 & 0.01 & 0.01 & 0.01\end{array}\right]^{T}$. It clearly shows that the system states are in irregular and unstable nonlinear vibrations. So the effective controller should be designed for the vibration inhibition and stable operation of the fractionalorder hydroturbine regulating system. 


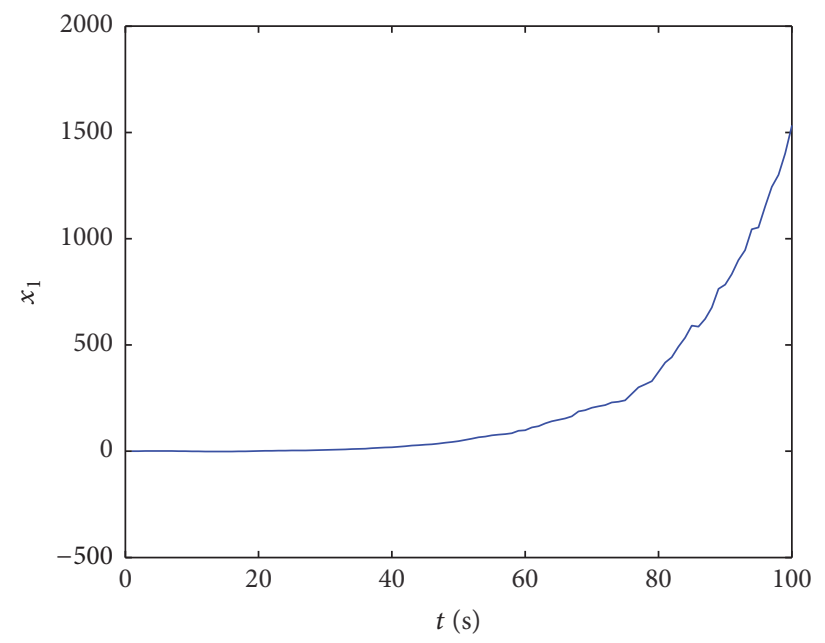

(a) State trajectory of $x_{1}$

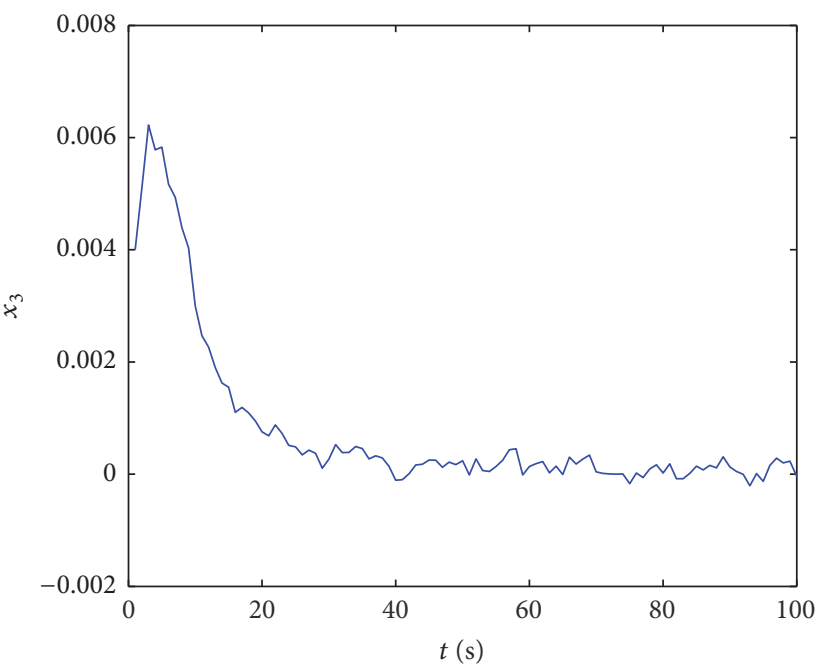

(c) State trajectory of $x_{3}$

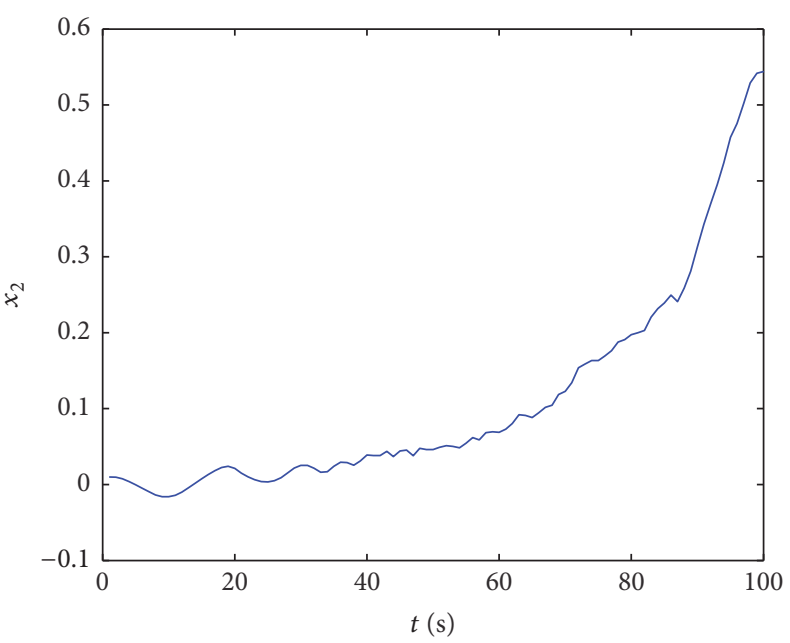

(b) State trajectory of $x_{2}$

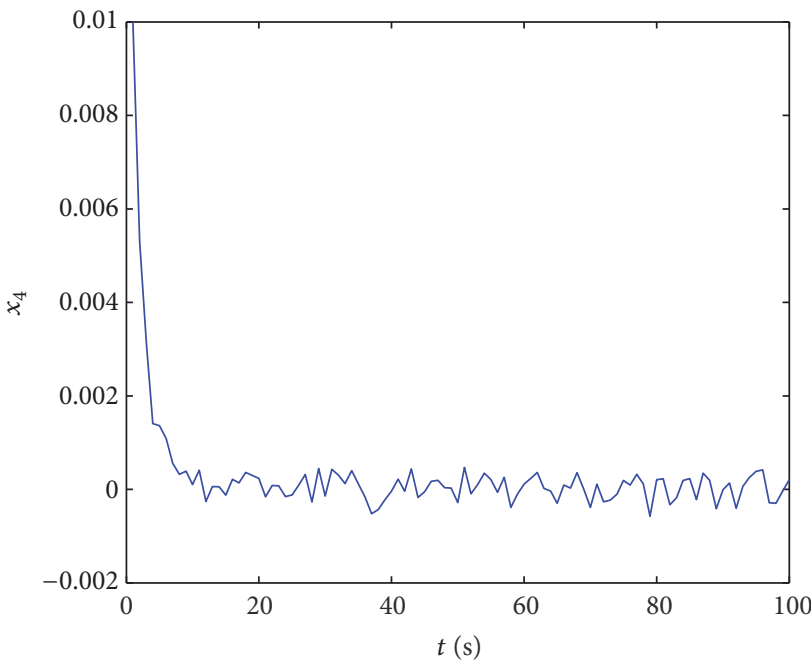

(d) State trajectory of $x_{4}$

FIGURE 1: State trajectories of fractional-order hydroturbine regulating system (4).

\section{Robust Fuzzy Controller Design}

3.1. Interval Matrix Theory. When the uncertain parameters are considered, the fractional-order hydroturbine regulating system could be rewritten as

$$
\begin{aligned}
\frac{d x_{1}}{d t}= & \tilde{a} x_{2}, \\
\frac{d x_{2}}{d t}= & \tilde{b} x_{3}-\tilde{c} x_{2}-\frac{1}{T_{a b}} \times \frac{E_{q}^{\prime} V_{s}}{x_{d \Sigma}^{\prime}} \sin x_{1}-\frac{1}{T_{a b}} \\
& \times \frac{V_{s}^{2}}{2} \frac{x_{d \Sigma}^{\prime}-x_{q \Sigma}}{x_{d \Sigma}^{\prime} x_{q \Sigma}} \sin 2 x_{1}, \\
\frac{d x_{3}}{d t}= & -\tilde{d} x_{3}+\tilde{e} x_{4}, \\
\frac{d^{\alpha} x_{4}}{d t^{\alpha}}= & \tilde{f} x_{4},
\end{aligned}
$$

where $\tilde{a} \in\left[a_{1}, a_{2}\right], \tilde{b} \in\left[b_{1}, b_{2}\right], \tilde{c} \in\left[c_{1}, c_{2}\right], \tilde{d} \in\left[d_{1}, d_{2}\right], \tilde{e} \in$ $\left[e_{1}, e_{2}\right]$, and $\tilde{f} \in\left[f_{1}, f_{2}\right]$.

To discuss the parameter uncertainties of the coefficient matrix of the fractional-order hydroturbine regulating system (5), the following interval matrix theory is introduced.

The linear fractional-order interval system is given as [31]

$$
\frac{d^{\alpha} x}{d t^{\alpha}}=\widetilde{A} x(t)
$$

where the interval uncertain matrix $\widetilde{A}$ satisfies

$\widetilde{A} \in N\left[A^{l}, A^{u}\right]=\left\{\widetilde{A} \in R^{n \times n} \mid a_{i j}^{l} \leq a_{i j} \leq a_{i j}^{u}, i, j=\right.$ $1, \ldots, n\}$, where $A^{l}$ and $A^{u}$ are the lower and upper bounds of matrix $\widetilde{A}$, respectively.

The matrix $\widetilde{A}$ could be equivalent presented as

$$
\widetilde{A}=A_{0 i}+E \Sigma F,
$$


where

$$
\begin{aligned}
A_{0 i} & =\frac{1}{2}\left(A_{i}^{l}+A_{i}^{u}\right), \\
\Sigma & \in \Sigma^{*}=\left\{\Sigma_{i} \in R^{n \times n} \mid \Sigma_{i}\right. \\
& =\operatorname{diag}\left(\varepsilon_{11}, \ldots, \varepsilon_{1 n}, \ldots, \varepsilon_{n 1}, \ldots, \varepsilon_{n n}\right),\left|\varepsilon_{i j}\right| \leq 1, i, j \\
& =1, \ldots, n\}, \\
E & =\left(\sqrt{h_{11}} e_{1}, \ldots, \sqrt{h_{1 n}} e_{1}, \ldots, \sqrt{h_{n 1}} e_{n}, \ldots, \sqrt{h_{n n}} e_{n}\right), \\
F & =\left(\sqrt{h_{11}} e_{1}, \ldots, \sqrt{h_{1 n}} e_{n}, \ldots, \sqrt{h_{n 1}} e_{1}, \ldots, \sqrt{h_{n n}} e_{n}\right)^{T},
\end{aligned}
$$

where $H=\left(h_{i j}\right)_{n \times n}=H_{i}=(1 / 2)\left(A_{i}^{u}-A_{i}^{l}\right), e_{i}(i=1, \ldots, n)$ is the $n \times n$ identity matrix $i$ th column.

Note that, for any $i$ and $\Sigma \in \Sigma^{*}$, there is

$$
\Sigma \Sigma^{T}=\Sigma^{T} \Sigma \leq I, \quad(I \text { is } n \times n \text { unit matrix }) .
$$

3.2. Parallel Distributed Compensation (PDC) Controller. Rule $R^{i}$ : IF $z_{1}(t)$ is $M_{i 1}$ and $\ldots$ and $z_{n}(t)$ is $M_{i p}$

$$
\begin{aligned}
\operatorname{THEN} \frac{d^{\alpha} x(t)}{d t^{\alpha}}= & \left(\widetilde{A}_{i}+\Delta A_{i}\right) x(t) \\
& +\left(B_{i}+\Delta B_{i}\right) u(t), \\
r & \quad r=(1,2, \ldots, r),
\end{aligned}
$$

where the fuzzy set is $M_{i j}(j=1,2, \ldots, n)$ and $r$ is the IFTHEN rules number, $x(t) \in R^{n}$ is the state vector, $A_{i} \in R^{n \times n}$, $z(t)=\left[z_{1}(t), z_{2}(t), \ldots, z_{p}(t)\right]$ are the premise variables, the fractional order is $\alpha(0<\alpha<1)$, and the control input is $u(t)$. The Takagi-Sugeno fuzzy model total output is introduced as

$$
\begin{aligned}
\frac{d^{\alpha} x}{d t^{\alpha}}= & \sum_{i=1}^{r} h_{i}(z(t))\left(A_{i}+\Delta A_{i}\right) x(t) \\
& +\sum_{i=1}^{r} h_{i}(z(t))\left(B_{i}+\Delta B_{i}\right) u(t),
\end{aligned}
$$

where

$$
\begin{aligned}
h_{i}(z(t)) & =\frac{\prod_{j=1}^{n} M_{i j}\left(z_{j}(t)\right)}{\sum_{i=1}^{r} \prod_{j=1}^{n} M_{i j}\left(z_{j}(t)\right)} \geq 0, \\
\sum_{i=1}^{r} h_{i}(z(t)) & =1 .
\end{aligned}
$$

The new fuzzy controller $u(t)$ is designed on the basis of parallel distributed compensation (PDC) and represented as follows.

Rule $R^{i}$ : IF $z_{1}(t)$ is $M_{i 1}$ and $\ldots$ and $z_{n}(t)$ is $M_{i p}$

$$
\text { THEN } u(t)=K_{i} x(t) \quad(i=1,2, \ldots, r)
$$

The parallel distributed compensation controller is shown as follows:

$$
u(t)=\sum_{i=1}^{r} h_{i}(z(t)) K_{i} x(t),
$$

where $K_{i}(i=1,2, \ldots, r)$ represents the feedback gain.

By substituting (14) to (11), there follows

$$
\begin{aligned}
\frac{d^{\alpha} x}{d t^{\alpha}}= & \sum_{i=1}^{r} h_{i}(z(t))\left(A_{i}+\Delta A_{i}\right) x(t) \\
& +\sum_{i=1}^{r} h_{i}(z(t))\left(B_{i}+\Delta B_{i}\right) \sum_{i=1}^{r} h_{i}(z(t)) K_{i} x(t) .
\end{aligned}
$$

According to the term $\sum_{i=1}^{r} h_{i}(z(t))=1$, (15) can be equally written as

$$
\begin{aligned}
& \frac{d^{\alpha} x}{d t^{\alpha}}=\sum_{i=1}^{r} \sum_{j=1}^{r} h_{i}(z(t)) h_{j}(z(t)) \\
& \cdot\left(A_{i}+\Delta A_{i}+\left(B_{i}+\Delta B_{i}\right) K_{j}\right) x(t) .
\end{aligned}
$$

3.3. Takagi-Sugeno Fuzzy Model of Fractional-Order Hydroturbine Regulating System. For the convenience of the coefficient matrix, the Maclaurin series expansion is introduced:

$$
\begin{aligned}
\sin x= & x-\frac{x^{3}}{3 !}+\frac{x^{5}}{5 !}-\frac{x^{7}}{7 !}+\cdots+(-1)^{n} \frac{x^{2 n+1}}{(2 n+1) !} \\
& +\cdots
\end{aligned}
$$

Assume $x_{2} \in[-d, d]$; here $d=2$. The Takagi-Sugeno fuzzy model is established, with the following two rules to describe the dynamic behavior of the system:

$$
\begin{aligned}
& R^{1}: \text { IF } x_{2} \text { is } M_{1}\left(x_{2}(t)\right) \text {, THEN } d^{\alpha} x(t) / d t^{\alpha}=\left(\widetilde{A}_{1}+\right. \\
& \left.\Delta A_{1}\right) x(t)+\left(B_{1}+\Delta B_{1}\right) u(t) ; \\
& R^{2}: \text { IF } x_{2} \text { is } M_{2}\left(x_{2}(t)\right), \text { THEN } d^{\alpha} x(t) / d t^{\alpha}=\left(\widetilde{A}_{2}+\right. \\
& \left.\Delta A_{2}\right) x(t)+\left(B_{2}+\Delta B_{2}\right) u(t) .
\end{aligned}
$$

The membership functions are taken as follows:

$$
\begin{aligned}
& M_{1}\left(x_{2}(t)\right)=\frac{1}{2}\left(1+\frac{x_{2}(t)}{d}\right), \\
& M_{2}\left(x_{2}(t)\right)=\frac{1}{2}\left(1-\frac{x_{2}(t)}{d}\right) .
\end{aligned}
$$

In view of the above description, there is

$$
\widetilde{A}_{1}=\left[\begin{array}{cccc}
0 & \tilde{a} & 0 & 0 \\
\frac{17231}{16951} & -\tilde{b} & \widetilde{c} & 0 \\
0 & 0 & -\tilde{d} & \widetilde{e} \\
0 & 0 & 0 & -\widetilde{f}
\end{array}\right] \text {, }
$$




$$
\begin{aligned}
& \widetilde{A}_{2}=\left[\begin{array}{cccc}
0 & \tilde{a} & 0 & 0 \\
\frac{1577}{16951} & -\tilde{b} & \widetilde{c} & 0 \\
0 & 0 & -\widetilde{d} & \tilde{e} \\
0 & 0 & 0 & -\tilde{f}
\end{array}\right], \\
& B_{1}=B_{2}=I_{4 \times 4} .
\end{aligned}
$$

So the fractional-order Takagi-Sugeno fuzzy model of the hydroturbine regulating system can be represented as

$$
\frac{d^{\alpha} x}{d t^{\alpha}}=\sum_{i=1}^{2} h_{i}(z(t))\left(\widetilde{A}_{i}+\Delta A_{i}+\left(B_{i}+\Delta B_{i}\right) u(t)\right) .
$$

3.4. Controller Design. According to the PDC law, one has

$$
u(t)=\sum_{j=1}^{2} h_{j}(z(t)) K_{j} x(t) .
$$

Then, (20) could be rewritten as

$$
\begin{aligned}
& \frac{d^{\alpha} x}{d t^{\alpha}}=\sum_{i=1}^{2} \sum_{j=1}^{2} h_{i}(x(t)) h_{j}(x(t)) \\
& \cdot\left(\widetilde{A}_{i}+\Delta A_{i}+\left(B_{i}+\Delta B_{i}\right) K_{j}\right) x(t) .
\end{aligned}
$$

With the help of interval matrix theory in Section 3.1, (22) can be equivalently given as

$$
\begin{aligned}
& \frac{d^{\alpha} x}{d t^{\alpha}}=\sum_{i=1}^{2} \sum_{j=1}^{2} h_{i}(x(t)) h_{j}(x(t)) \\
& \cdot\left(A_{0 i}+E \Sigma F+\Delta A_{i}+\left(B_{i}+\Delta B_{i}\right) K_{j}\right) x(t) .
\end{aligned}
$$

Assumption 1. The parameter uncertainties considered here are norm-bounded in the following form:

$$
\begin{aligned}
\Delta A_{i} & =D_{i} F_{i}(t) E_{1 i}, \\
\Delta B_{i} & =D_{i} F_{i}(t) E_{2 i},
\end{aligned}
$$

where $D_{i}, E_{1 i}, E_{2 i}$ are known real constant matrices of appropriate dimensions and $F_{i}$ is a diagonal random matrix with Lebesgue-measurable elements and satisfies $F_{i} F_{i}^{T} \leq I$; $I$ is the identity matrix with an appropriate dimension.

According to Assumption 1, (23) can be equally written as

$$
\begin{aligned}
& \frac{d^{\alpha} x}{d t^{\alpha}}=\sum_{i=1}^{2} \sum_{j=1}^{2} h_{i}(x(t)) h_{j}(x(t)) \\
& \cdot\left(A_{0 i}+E \Sigma F+D_{i} F_{i} E_{1 i}+\left(B_{i}+D_{i} F_{i} E_{2 i}\right) K_{j}\right) x(t) .
\end{aligned}
$$

Lemma 2 (see [32]). For the linear fractional-order system presented below,

$$
\begin{aligned}
& D^{\alpha} x=A x, \\
& x(0)=x_{0},
\end{aligned}
$$

where $A \in R^{n \times n}, x \in R^{n}$, and $\alpha=\left[\alpha_{1}, \alpha_{2}, \ldots, \alpha_{i}, \ldots, \alpha_{n}\right](0<$ $\left.\alpha_{i} \leq 1\right)$, for all eigenvalues $\lambda_{i}$ of matrix $A$, when and only when $\left|\arg \left(\lambda_{i}\right)\right|>\alpha \pi / 2$ is satisfied; system (26) is asymptotically stable.

Theorem 3. Assume the system matrix A meets the Lyapunov function; that is, there is real positive definite symmetric matrix $P$ as well as semipositive definite matrix $Q$ meeting $A^{T} P+P A=$ $-Q$. The fractional-order hydroturbine regulating system (25) then will converge to the equilibrium point asymptotically.

Proof. Assume $\lambda$ is an eigenvalue of the system matrix $A$ and the eigenvector is $\xi$; one can easily get

$$
A \xi=\lambda \xi
$$

For both sides of (27), making conjugation and transpose, one has

$$
\overline{(A \xi)}^{T}=\bar{\lambda} \xi^{T}
$$

For (27) left side, multiplying $\xi^{T} P$, one can be obtain

$$
\xi^{T} P A \xi=\lambda \xi^{T} P \xi
$$

For (28) right side, making the similar treatment and multiplying $P \xi$, one can also get

$$
\overline{(A \xi)}^{T} P \xi=\bar{\lambda} \xi^{T} P \xi .
$$

Combining (29) and (30), one can easily get

$$
\xi^{T}\left(P A+A^{T} P\right) \xi=(\lambda+\bar{\lambda}) \xi^{T} P \xi .
$$

According to Theorem $3, A^{T} P+P A$ is a seminegative definite matrix. So, for any nonzero vector $\xi$, one has

$$
\begin{gathered}
\xi^{T}\left(P A+A^{T} P\right) \xi=\xi^{T}(-Q) \xi \leq 0, \\
\xi^{T} P \xi>0 .
\end{gathered}
$$

Combining (31) and (32), one can easily get

$$
(\lambda+\bar{\lambda})=\xi^{T}(-Q) \overline{\xi \xi^{T} P \xi} \leq 0 .
$$

It means, for all eigenvalues of matrix $A$, there is

$$
|\arg (\lambda)| \geq \frac{\pi}{2}>\frac{\alpha \pi}{2}, \quad(\alpha<1)
$$

Referring to Lemma 2, the fractional-order hydroturbine regulating system (25) then will converge to the equilibrium point asymptotically. The proof is complete.

The following more flexible theorem is presented on the basis of Theorem 3 .

Theorem 4. For any system variables $x=\left[x_{1}, x_{2}, \ldots, x_{n}\right]^{T}$, there exists a real positive definite symmetric matrix $P$ meeting $J=x^{T} P\left(d^{\alpha} x / d t^{\alpha}\right) \leq 0,\left(x^{T} P\left(d^{\alpha} x / d t^{\alpha}\right)\right.$ is called $J$ function $)$. The fractional-order hydroturbine regulating system (25) then 
will converge to the equilibrium point asymptotically. The condition $J=x^{T} P\left(d^{\alpha} x / d t^{\alpha}\right) \leq 0$ could be equivalently given as

$$
J_{0}=x^{T} P \frac{d^{\alpha} x}{d t^{\alpha}}+\left(\frac{d^{\alpha} x}{d t^{\alpha}}\right)^{T} P x \leq 0 .
$$

Proof. From Theorem 3, there is

$$
A^{T} P+P A=-Q
$$

According to (36), one can easily get

$$
x^{T}\left(A^{T} P+P A\right) x=-x^{T} Q x
$$

where $Q$ is a semipositive definite matrix. That is to say, for any system variable $x$, there is

$$
x^{T}\left(A^{T} P+P A\right) x=-x^{T} Q x \leq 0 .
$$

Substituting (26) to (38), one has

$$
x^{T} P \frac{d^{\alpha} x}{d t^{\alpha}}+\left(\frac{d^{\alpha} x}{d t^{\alpha}}\right)^{T} P x \leq 0 .
$$

The positive definite symmetric matrix $P$ is supposed as

$$
P=\left[\begin{array}{cccc}
a_{11} & a_{12} & \cdots & a_{1 n} \\
a_{21} & a_{22} & \cdots & a_{2 n} \\
\vdots & \vdots & \ddots & \vdots \\
a_{n 1} & a_{n 2} & \cdots & a_{n n}
\end{array}\right]
$$

Introducing (40) to (39), one gets

$$
\sum_{i, j=1}^{n} a_{i j} x_{i} \frac{d^{\alpha} x_{j}}{d t^{\alpha}}+\sum_{i, j=1}^{n} a_{i j} x_{j} \frac{d^{\alpha} x_{i}}{d t^{\alpha}} \leq 0
$$

For $a_{i j}=a_{j i}(\forall i, j),(41)$ could be rewritten as

$$
\begin{aligned}
& \sum_{i, j=1}^{n} a_{i j} x_{i} \frac{d^{\alpha} x_{j}}{d t^{\alpha}}+\sum_{i, j=1}^{n} a_{i j} x_{j} \frac{d^{\alpha} x_{i}}{d t^{\alpha}}=2 \sum_{i, j=1}^{n} a_{i j} x_{j} \frac{d^{\alpha} x_{i}}{d t^{\alpha}} \\
& =2 x^{T} P \frac{d^{\alpha} x}{d t^{\alpha}}=2\left(\frac{d^{\alpha} x}{d t^{\alpha}}\right)^{T} P x \leq 0 .
\end{aligned}
$$

It is clear that Theorem 4 is equivalent to Theorem 3 . The proof is finished.

Based on the above theorems, the more practical stability conditions are proposed as follows.

Theorem 5. The fractional-order hydroturbine regulating system (25) then will converge to the equilibrium point asymptotically, once there exist a plus constant $\eta$, a positive definite matrix $P$, and the controller gain matrices $K_{i}(i=1,2)$ which satisfy the following LMIs:

$$
\begin{gathered}
{\left[\begin{array}{cc}
\Phi_{i i} & Q F^{T} \\
F Q & \eta^{-1} I
\end{array}\right]<0,} \\
{\left[\begin{array}{cc}
\Phi_{i j} & Q F^{T} \\
F Q & -\eta^{-1} I
\end{array}\right]<0,} \\
Q>0,
\end{gathered}
$$

where

$$
\begin{aligned}
\Phi_{i i}= & Q\left(A_{i}+D_{i} F_{i} E_{1 i}\right)^{T}+M_{i}^{T}\left(B_{i}+D_{i} F_{i} E_{2 i}\right)^{T} \\
& +\left(A_{i}+D_{i} F_{i} E_{1 i}\right) Q+\left(B_{i}+D_{i} F_{i} E_{2 i}\right) M_{i} \\
& +\eta E E^{T}, \\
\Phi_{i j}= & Q\left(A_{i}+D_{i} F_{i} E_{1 i}\right)^{T}+M_{j}^{T}\left(B_{i}+D_{i} F_{i} E_{2 i}\right)^{T} \\
& +Q\left(A_{j}+D_{j} F_{j} E_{1 j}\right)^{T} \\
& +M_{i}^{T}\left(B_{j}+D_{j} F_{j} E_{2 j}\right)^{T}+\left(A_{i}+D_{i} F_{i} E_{1 i}\right) Q \\
& +\left(B_{i}+D_{i} F_{i} E_{2 i}\right) M_{j}+\left(A_{j}+D_{j} F_{j} E_{1 j}\right) Q \\
& +\left(B_{j}+D_{j} F_{j} E_{2 j}\right) M_{i}+\eta E E^{T},
\end{aligned}
$$

$Q=P^{-1}, M_{i}=K_{i} P^{-1}, M_{j}=K_{j} P^{-1}$, and $I$ is $4 \times 4$ unit matrix.

Proof. Based on Theorem 4, choose $J_{0}=x^{T} P\left(d^{\alpha} x / d t^{\alpha}\right)+$ $\left(d^{\alpha} x / d t^{\alpha}\right)^{T} P x$ as $J$ function for system (25).

$$
\begin{aligned}
J_{0} & =x^{T} P \frac{d^{\alpha} x}{d t^{\alpha}}+\left(\frac{d^{\alpha} x}{d t^{\alpha}}\right)^{T} P x=\sum_{i=1}^{4} \sum_{j=1}^{4} h_{i} h_{j}\left(A_{0 i}+E \Sigma F+D_{i} F_{i} E_{1 i}+\left(B_{i}+D_{i} F_{i} E_{2 i}\right) K_{j}\right)^{T} x^{T} P x+x^{T} P \sum_{i=1}^{4} \sum_{j=1}^{4} h_{i} h_{j}\left(A_{0 i}\right. \\
& \left.+E \Sigma F+D_{i} F_{i} E_{1 i}+\left(B_{i}+D_{i} F_{i} E_{2 i}\right) K_{j}\right)=\sum_{i=1}^{4} \sum_{j=1}^{4} h_{i} h_{j} x^{T}\left\{\left[\left(A_{0 i}+D_{i} F_{i} E_{1 i}+\left(B_{i}+D_{i} F_{i} E_{2 i}\right) K_{j}\right)^{T} P+F^{T} \Sigma E^{T} P\right]\right.
\end{aligned}
$$




$$
\begin{aligned}
& \left.+\left[P\left(A_{0 i}+D_{i} F_{i} E_{1 i}+\left(B_{i}+D_{i} F_{i} E_{2 i}\right) K_{j}\right)+P E \Sigma F\right] x\right\}=\sum_{i=1}^{4} h_{i}^{2} x^{T}\left[\left(A_{0 i}+D_{i} F_{i} E_{1 i}+\left(B_{i}+D_{i} F_{i} E_{2 i}\right) K_{i}\right)^{T} P+F^{T} \Sigma E^{T} P\right. \\
& \left.+P\left(A_{0 i}+D_{i} F_{i} E_{1 i}+\left(B_{i}+D_{i} F_{i} E_{2 i}\right) K_{i}\right)+P E \Sigma F\right] x+\sum_{i<j}^{4} h_{i} h_{j} x^{T}\left[\left(A_{0 i}+D_{i} F_{i} E_{1 i}+\left(B_{i}+D_{i} F_{i} E_{2 i}\right) K_{j}\right)^{T} P+F^{T} \Sigma E^{T} P\right. \\
& \left.+P\left(A_{0 i}+D_{i} F_{i} E_{1 i}+\left(B_{i}+D_{i} F_{i} E_{2 i}\right) K_{j}\right)+P E \Sigma F\right] x+\sum_{i>j}^{4} h_{i} h_{j} x^{T}\left[\left(A_{0 j}+D_{j} F_{j} E_{1 j}+\left(B_{j}+D_{j} F_{j} E_{2 j}\right) K_{i}\right)^{T} P\right. \\
& \left.+F^{T} \Sigma E^{T} P+P\left(A_{0 j}+D_{j} F_{j} E_{1 j}+\left(B_{j}+D_{j} F_{j} E_{2 j}\right) K_{i}\right)+P E \Sigma F\right] x=\sum_{i=1}^{4} h_{i}^{2} x^{T}\left[\left(A_{0 i}+D_{i} F_{i} E_{1 i}+\left(B_{i}+D_{i} F_{i} E_{2 i}\right) K_{i}\right)^{T} P\right. \\
& \left.+P\left(A_{0 i}+D_{i} F_{i} E_{1 i}+\left(B_{i}+D_{i} F_{i} E_{2 i}\right) K_{i}\right)\right] x+\sum_{i=1}^{4} h_{i}^{2} x^{T}\left(F^{T} \Sigma E^{T} P+P E \Sigma F\right) x+2 \sum_{i<j}^{4} h_{i} h_{j} x^{T}\left(F^{T} \Sigma E^{T} P+P E \Sigma F\right) x \\
& +\sum_{i<j}^{4} h_{i} h_{j} x^{T}\left[\left(A_{0 i}+D_{i} F_{i} E_{1 i}+\left(B_{i}+D_{i} F_{i} E_{2 i}\right) K_{j}\right)^{T} P+P\left(A_{0 i}+D_{i} F_{i} E_{1 i}+\left(B_{i}+D_{i} F_{i} E_{2 i}\right) K_{j}\right)\right] x+\sum_{i>j}^{4} h_{i} h_{j} x^{T}\left[\left(A_{0 j}\right.\right. \\
& \left.\left.+D_{j} F_{j} E_{1 j}+\left(B_{j}+D_{j} F_{j} E_{2 j}\right) K_{i}\right)^{T} P+P\left(A_{0 j}+D_{j} F_{j} E_{1 j}+\left(B_{j}+D_{j} F_{j} E_{2 j}\right) K_{i}\right)\right] x .
\end{aligned}
$$

Considering that $\sum_{i=1}^{4} h_{i}^{2}+2 \sum_{i<j}^{4} h_{i} h_{j}=1$ and

$$
\begin{aligned}
& \sum_{i<j}^{4} h_{i} h_{j} x^{T}\left[\left(A_{0 i}+D_{i} F_{i} E_{1 i}+\left(B_{i}+D_{i} F_{i} E_{2 i}\right) K_{j}\right)^{T} P+P\left(A_{0 i}+D_{i} F_{i} E_{1 i}+\left(B_{i}+D_{i} F_{i} E_{2 i}\right) K_{j}\right)\right] x \\
& \quad+\sum_{i>j}^{4} h_{i} h_{j} x^{T}\left[\left(A_{0 j}+D_{j} F_{j} E_{1 j}+\left(B_{j}+D_{j} F_{j} E_{2 j}\right) K_{i}\right)^{T} P+P\left(A_{0 j}+D_{j} F_{j} E_{1 j}+\left(B_{j}+D_{j} F_{j} E_{2 j}\right) K_{i}\right)\right] x \\
& =\sum_{i<j}^{4} h_{i} h_{j} x^{T}\left\{\left[\left(A_{0 i}+D_{i} F_{i} E_{1 i}+\left(B_{i}+D_{i} F_{i} E_{2 i}\right) K_{j}\right)+\left(A_{0 j}+D_{j} F_{j} E_{1 j}+\left(B_{j}+D_{j} F_{j} E_{2 j}\right) K_{i}\right)\right]^{T} P\right. \\
& \left.+P\left[\left(A_{0 i}+D_{i} F_{i} E_{1 i}+\left(B_{i}+D_{i} F_{i} E_{2 i}\right) K_{j}\right)+\left(A_{0 j}+D_{j} F_{j} E_{1 j}+\left(B_{j}+D_{j} F_{j} E_{2 j}\right) K_{i}\right)\right]\right\} x \\
& \quad=2 \sum_{i<j}^{4} h_{i} h_{j} x^{T}\left\{\left[\frac{\left(A_{0 i}+D_{i} F_{i} E_{1 i}+\left(B_{i}+D_{i} F_{i} E_{2 i}\right) K_{j}\right)+\left(A_{0 j}+D_{j} F_{j} E_{1 j}+\left(B_{j}+D_{j} F_{j} E_{2 j}\right) K_{i}\right)}{2}\right]^{T} P\right. \\
& \left.\quad+P\left[\frac{\left(A_{0 i}+D_{i} F_{i} E_{1 i}+\left(B_{i}+D_{i} F_{i} E_{2 i}\right) K_{j}\right)+\left(A_{0 j}+D_{j} F_{j} E_{1 j}+\left(B_{j}+D_{j} F_{j} E_{2 j}\right) K_{i}\right)}{2}\right]\right\} x,
\end{aligned}
$$

select

$$
\begin{aligned}
& G_{i i}=A_{0 i}+D_{i} F_{i} E_{1 i}+\left(B_{i}+D_{i} F_{i} E_{1 i}\right) K_{i}, \\
& G_{i j}=\frac{\left(A_{0 i}+D_{i} F_{i} E_{1 i}+\left(B_{i}+D_{i} F_{i} E_{1 i}\right) K_{j}\right)+\left(A_{0 j}+D_{j} F_{j} E_{1 j}+\left(B_{j}+D_{j} F_{j} E_{2 j}\right) K_{i}\right)}{2} .
\end{aligned}
$$


There follows

$$
\begin{aligned}
J_{0}= & \sum_{i=1}^{4} h_{i}^{2} x^{T}\left(G_{i i}^{T} P+P G_{i i}\right) x \\
& +2 \sum_{i<j}^{4} h_{i} h_{j} x^{T}\left(G_{i j}^{T} P+P G_{i j}\right) x+x^{T} F^{T} \Sigma E^{T} P x \\
& +x^{T} P E \Sigma F x .
\end{aligned}
$$

Select $\xi^{T}=x^{T} P E, \theta=F x$, and there is

$$
2 x^{T} P E \Sigma F x \leq \eta x^{T} P E E^{T} P x+\eta^{-1} x^{T} F^{T} F x .
$$

Taking the transpose of both sides in (49), one obtains

$$
2 x^{T} F^{T} \Sigma E^{T} P x \leq \eta x^{T} P E E^{T} P x+\eta^{-1} x^{T} F^{T} F x .
$$

According to (50) and (49), one gets

$$
\begin{aligned}
& x^{T} F^{T} \sum E^{T} P x+x^{T} P E \Sigma F x \\
& \quad \leq \eta x^{T} P E E^{T} P x+\eta^{-1} x^{T} F^{T} F x .
\end{aligned}
$$

By substituting (51) into (48), one has

$$
\begin{aligned}
J_{0} \leq & \sum_{i=1}^{4} h_{i}^{2} x^{T}\left(G_{i i}^{T} P+P G_{i i}\right) x \\
& +2 \sum_{i<j}^{4} h_{i} h_{j} x^{T}\left(G_{i j}^{T} P+P G_{i j}\right) x+\eta x^{T} P E E^{T} P x \\
& +\eta^{-1} x^{T} F^{T} F x .
\end{aligned}
$$

Note that $\sum_{i=1}^{4} h_{i}^{2}+2 \sum_{i<j}^{4} h_{i} h_{j}=1$, so (52) can be equally represented as

$$
\begin{aligned}
J_{0} & \leq \sum_{i=1}^{4} h_{i}^{2} x^{T}\left(G_{i i}^{T} P+P G_{i i}+\eta P E E^{T} P+\eta^{-1} F^{T} F\right) x \\
& +2 \sum_{i<j}^{4} h_{i} h_{j} x^{T}\left(G_{i j}^{T} P+P G_{i j}+\eta P E E^{T} P+\eta^{-1} F^{T} F\right) \\
& \cdot x
\end{aligned}
$$

Accordingly, (25) can be assured as long as the following inequalities hold.

$$
\begin{array}{ll}
G_{i i}^{T} P+P G_{i i}+\eta P E E^{T} P+\eta^{-1} F^{T} F<0 & (i, j=1,2), \\
G_{i j}^{T} P+P G_{i j}+\eta P E E^{T} P+\eta^{-1} F^{T} F<0 & (i<j \leq 2) .
\end{array}
$$

It is clear that the fractional-order hydroturbine regulating system (25) then will converge to the equilibrium point asymptotically once (54) are satisfied.

With the help of Schur's theorem [33], one can easily transform (54) to the standard form of linear matrix inequalities, which are presented as (43). The proof is complete.

\section{Numerical Simulations}

Considering the fractional-order hydroturbine regulating system (4) with uncertain parameters, $\widetilde{a}=314+0.1 \sin (t)$, $\widetilde{b}=2 / 9+0.1 \cos (t), \tilde{c}=1 / 9+0.1 \sin (t), \widetilde{d}=5 / 2+0.1 \cos (t)$, $\widetilde{e}=33 / 2+0.1 \sin (t)$, and $\widetilde{f}=10+0.1 \cos (t)$.

Therefore, $\tilde{a} \in[313.9,314.1], \tilde{b} \in[11 / 90,29 / 90], \tilde{c} \in$ $[1 / 90,19 / 90], \widetilde{d} \in[2.4,2.6], \widetilde{e} \in[16.4,16.6]$, and $\widetilde{f} \in$ $[9.9,10.1]$.

To take control of the fractional-order hydroturbine regulating system with uncertain parameters, we take $d=2$, $A_{0 i}(i=1,2)$, and $E$ and $F$ can be calculated. The value of $\Sigma$ can also refer to Section 3.1.

$$
\begin{aligned}
& B_{1}=B_{2}=I_{4 \times 4}, \\
& D_{1}=D_{2}=E_{11}=E_{12}=E_{21}=E_{22}=I_{4 \times 4}, \\
& F_{1}=F_{2}=\operatorname{diag}(\operatorname{diag}(\operatorname{rand}(4,4))) .
\end{aligned}
$$

According to the above theorems and $\eta=1000$, in the environment of Matlab 7.0 LMI toolbox we acquire

$$
P=10^{-4} \times\left[\begin{array}{cccc}
0.0000 & -0.0000 & 0.0000 & -0.0000 \\
-0.0000 & 0.4004 & 0.0013 & -0.0004 \\
0.0000 & 0.0013 & 0.4955 & 0.0009 \\
-0.0000 & 0.0004 & 0.0093 & 0.4965
\end{array}\right]
$$$$
K_{1}=10^{3}
$$

$$
\begin{gathered}
\times\left[\begin{array}{cccc}
-0.0007 & -7.4759 & -0.0232 & 0.0079 \\
-0.0003 & -0.0038 & -0.0000 & 0.0000 \\
0.0000 & 0.0000 & -0.0011 & -0.0072 \\
-0.0000 & -0.0000 & -0.0070 & 0.0052
\end{array}\right], \\
K_{2}=\left[\begin{array}{cccc}
-0.4730 & -797.0103 & -2.1527 & -0.7156 \\
-0.0270 & -4.2479 & -0.0467 & 0.0051 \\
0.0000 & 0.0372 & -1.0621 & -7.2386 \\
-0.0000 & -0.0049 & -7.0022 & 5.2363
\end{array}\right] .
\end{gathered}
$$

Figure 2 shows the simulation results of the traditional PID control method as well as the proposed scheme in this paper. It is clear that when the controller is applied, the system state variables quickly converge to zero point, which verifies the effectiveness. Compared with the traditional PID control method, it needs shorter transition time and the overshoot is more smaller than the designed scheme, which shows the superiority and good robustness of the proposed method.

\section{Conclusions}

The robust fuzzy control for fractional-order hydroturbine regulating system was studied in this paper. First, mathematical model of the fractional-order hydroturbine regulating system with uncertain parameters and random disturbances 

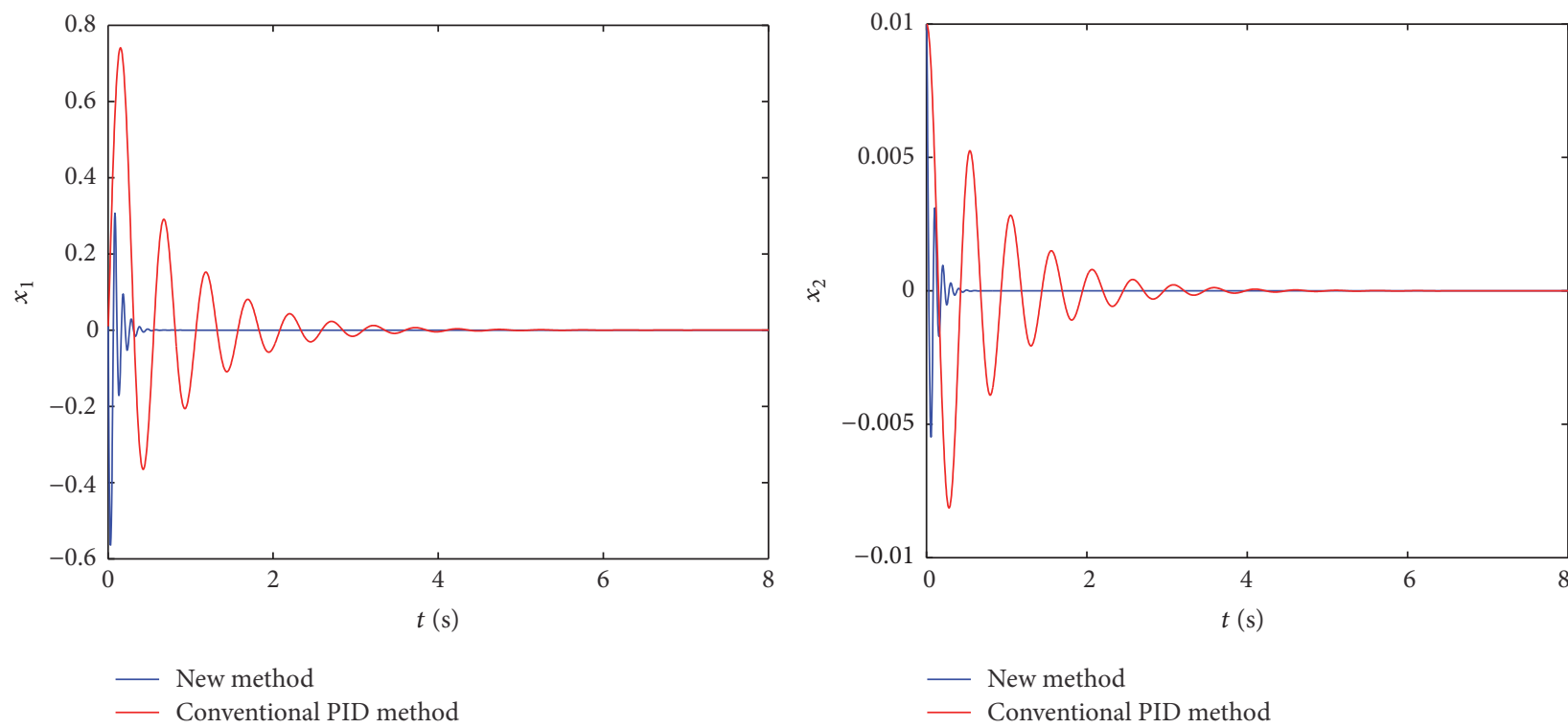

(a) State trajectory of $x_{1}$

(b) State trajectory of $x_{2}$
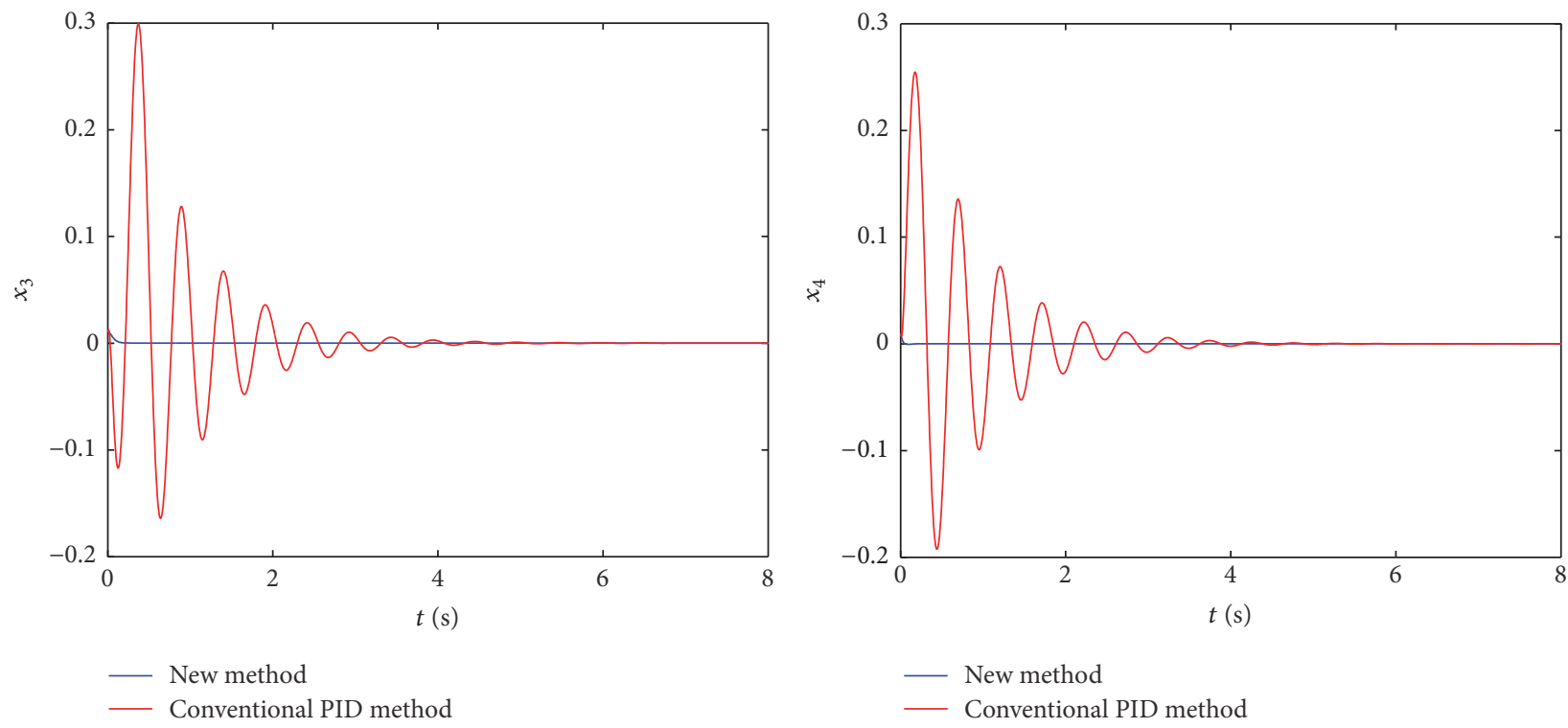

(d) State trajectory of $x_{4}$

(c) State trajectory of $x_{3}$

FIGURE 2: State trajectories of controlled fractional-order hydroturbine regulating system (4).

was introduced. Second, a fuzzy control method was proposed for fractional-order hydroturbine regulating system. Furthermore, the stability condition of the fractional-order hydroturbine regulating system was given as a group of linear matrix inequalities and the detailed mathematical proofs were presented. Besides, the method could handle the case of time-varying parameters and random disturbances, which has shown the good robustness. Finally, the validity and superiority were verified by the simulation results.

The scheme designed is simple and easy to implement and could be applied to similar fractional-order nonlinear systems. In the future, we will consider and extend the approach to other fractional-order hydroturbine governing systems, such as hydropower systems with time delay.

\section{Competing Interests}

The authors declare that there are no competing interests regarding the publication of this paper.

\section{Acknowledgments}

This work was supported by the Scientific Research Foundation of the National Natural Science Foundation (51509210 and 51479173), the Science and Technology Project of Shaanxi Provincial Water Resources Department (Grant no. 2015slkj11), the 111 Project from the Ministry of Education of China (B12007), and Yangling Demonstration Zone Technology Project (2014NY-32). 


\section{References}

[1] C. Vargas-De-León, "Volterra-type Lyapunov functions for fractional-order epidemic systems," Communications in Nonlinear Science and Numerical Simulation, vol. 24, no. 1-3, pp. 75-85, 2015.

[2] J. M. Yu, H. Hu, S. B. Zhou, and X. R. Lin, "Generalized MittagLeffler stability of multi-variables fractional order nonlinear systems," Automatica, vol. 49, no. 6, pp. 1798-1803, 2013.

[3] Z.-X. Zou, K. L. Zhou, Z. Wang, and M. Cheng, "Frequencyadaptive fractional-order repetitive control of shunt active power filters," IEEE Transactions on Industrial Electronics, vol. 62, no. 3, pp. 1659-1668, 2015.

[4] J. W. Zhu, D. Y. Chen, H. Zhao, and R. F. Ma, "Nonlinear dynamic analysis and modeling of fractional permanent magnet synchronous motors," Journal of Vibration and Control, vol. 22, no. 7, pp. 1855-1875, 2016.

[5] S. K. Luo and L. Li, "Fractional generalized Hamiltonian mechanics and Poisson conservation law in terms of combined Riesz derivatives," Nonlinear Dynamics, vol. 73, no. 1-2, pp. 639647, 2013.

[6] A. Flores-Tlacuahuac and L. T. Biegler, "Optimization of fractional order dynamic chemical processing systems," Industrial \& Engineering Chemistry Research, vol. 53, no. 13, pp. 5110-5127, 2014.

[7] P. Pennacchi, S. Chatterton, and A. Vania, "Modeling of the dynamic response of a Francis turbine," Mechanical Systems and Signal Processing, vol. 29, pp. 107-119, 2012.

[8] H. Zhang, D. Y. Chen, B. B. Xu, and F. F. Wang, "Nonlinear modeling and dynamic analysis of hydro-turbine governing system in the process of load rejection transient," Energy Conversion and Management, vol. 90, pp. 128-137, 2015.

[9] C. S. Li, J. Z. Zhou, J. Xiao, and H. Xiao, "Hydraulic turbine governing system identification using T-S fuzzy model optimized by chaotic gravitational search algorithm," Engineering Applications of Artificial Intelligence, vol. 26, no. 9, pp. 20732082, 2013.

[10] D. J. Ling and Y. Tao, "An analysis of the Hopf bifurcation in a hydroturbine governing system with saturation," IEEE Transactions on Energy Conversion, vol. 21, no. 2, pp. 512-515, 2006.

[11] X. D. Lai, Y. Zhu, G. L. Liao, X. Zhang, T. Wang, and W. B. Zhang, "Lateral vibration response analysis on shaft system of hydro turbine generator unit," Advances in Vibration Engineering, vol. 12, no. 6, pp. 511-524, 2013.

[12] Y. Xu, Z. H. Li, and X. D. Lai, "Dynamic model for hydro-turbine generator units based on a database method for guide bearings," Shock and Vibration, vol. 20, no. 3, pp. 411-421, 2013.

[13] Y. Zeng, L. X. Zhang, Y. K. Guo, J. Qian, and C. L. Zhang, “The generalized Hamiltonian model for the shafting transient analysis of the hydro turbine generating sets," Nonlinear Dynamics, vol. 76, no. 4, pp. 1921-1933, 2014.

[14] H. H. Li, D. Y. Chen, H. Zhang, F. Wang, and D. Ba, "Nonlinear modeling and dynamic analysis of a hydro-turbine governing system in the process of sudden load increase transient," Mechanical Systems and Signal Processing, vol. 80, pp. 414-428, 2016.

[15] W. C. Guo, J. D. Yang, M. J. Wang, and X. Lai, "Nonlinear modeling and stability analysis of hydro-turbine governing system with sloping ceiling tailrace tunnel under load disturbance," Energy Conversion and Management, vol. 106, pp. 127-138, 2015.
[16] L. Liu, W. Ding, C. X. Liu, H. G. Ji, and C. Q. Cao, "Hyperchaos synchronization of fractional-order arbitrary dimensional dynamical systems via modified sliding mode control," Nonlinear Dynamics, vol. 76, no. 4, pp. 2059-2071, 2014.

[17] A. Rhouma, F. Bouani, B. Bouzouita, and M. Ksouri, "Model predictive control of fractional order systems," Journal of Computational and Nonlinear Dynamics, vol. 9, no. 3, Article ID 031011, 2014.

[18] N. I. Chaudhary, M. A. Z. Raja, and A. U. Rehman Khan, "Design of modified fractional adaptive strategies for Hammerstein nonlinear control autoregressive systems," Nonlinear Dynamics, vol. 82, no. 4, pp. 1811-1830, 2015.

[19] G.-S. Wang, J.-W. Xiao, Y.-W. Wang, and J.-W. Yi, "Adaptive pinning cluster synchronization of fractional-order complex dynamical networks," Applied Mathematics and Computation, vol. 231, pp. 347-356, 2014.

[20] T. Takagi and M. Sugeno, "Fuzzy identification of systems and its applications to modeling and control," IEEE Transactions on Systems, Man and Cybernetics, vol. 15, no. 1, pp. 116-132, 1985.

[21] B. Wang, J.-Y. Xue, H.-Y. He, and D.-L. Zhu, "Analysis on a class of double-wing chaotic system and its control via linear matrix inequality," Acta Physica Sinica, vol. 63, no. 21, Article ID 210502, 2014.

[22] P. Balasubramaniam and S. Muralisankar, "Existence and uniqueness of fuzzy solution for semilinear fuzzy integro differential equations with nonlocal conditions," Computers \& Mathematics with Applications, vol. 47, no. 6-7, pp. 1115-1122, 2004.

[23] I. Elishakoff and M. Ohsaki, Optimization and Anti-Optimization of Structures under Uncertainty, Imperial College Press, London, UK, 2010.

[24] Y. Q. Chen, H.-S. Ahn, and D. Y. Xue, "Robust controllability of interval fractional order linear time invariant systems," Signal Processing, vol. 86, no. 10, pp. 2794-2802, 2006.

[25] H.-S. Ahn, Y. Q. Chen, and I. Podlubny, "Robust stability test of a class of linear time-invariant interval fractional-order system using Lyapunov inequality," Applied Mathematics and Computation, vol. 187, no. 1, pp. 27-34, 2007.

[26] D. H. Lee and Y. H. Joo, "On the generalized local stability and local stabilization conditions for discrete-time takagi-sugeno fuzzy systems," IEEE Transactions on Fuzzy Systems, vol. 22, no. 6, pp. 1654-1668, 2014.

[27] H. Y. Li, C. W. Wu, and Z. G. Feng, "Fuzzy dynamic outputfeedback control of non-linear networked discrete-time system with missing measurements," IET Control Theory \& Applications, vol. 9, no. 3, pp. 327-335, 2015.

[28] F. B. Li, P. Shi, L. G. Wu, and X. Zhang, "Fuzzy-model-based D-stability and nonfragile control for discrete-time descriptor systems with multiple delays," IEEE Transactions on Fuzzy Systems, vol. 22, no. 4, pp. 1019-1025, 2014.

[29] H. Yousef, "Design of adaptive fuzzy-based tracking control of input time delay nonlinear systems," Nonlinear Dynamics, vol. 79, no. 1, pp. 417-426, 2015.

[30] D. J. Ling, Bifurcation and Chaos of Hydraulic Turbine Governor, Hohai University, Nanjing, China, 2007.

[31] J.-G. Lu and Y.-Q. Chen, "Robust stability and stabilization of fractional-order interval systems with the fractional order $\alpha$ : the $0<\alpha<1$ case," IEEE Transactions on Automatic Control, vol. 55, no. 1, pp. 152-158, 2010. 
[32] D. Matignon, "Stability results for fractional differential equations with applications to control processing," Computational Engineering in Systems and Application, vol. 2, pp. 963-968, 1996.

[33] J. Z. Liu, Z. J. Huang, and J. Zhang, "The dominant degree and disc theorem for the Schur complement of matrix," Applied Mathematics and Computation, vol. 215, no. 12, pp. 4055-4066, 2010. 


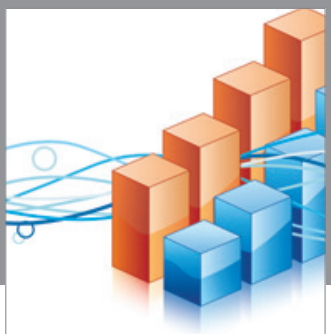

Advances in

Operations Research

vatem alat4

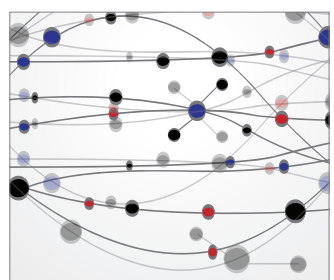

\section{The Scientific} World Journal
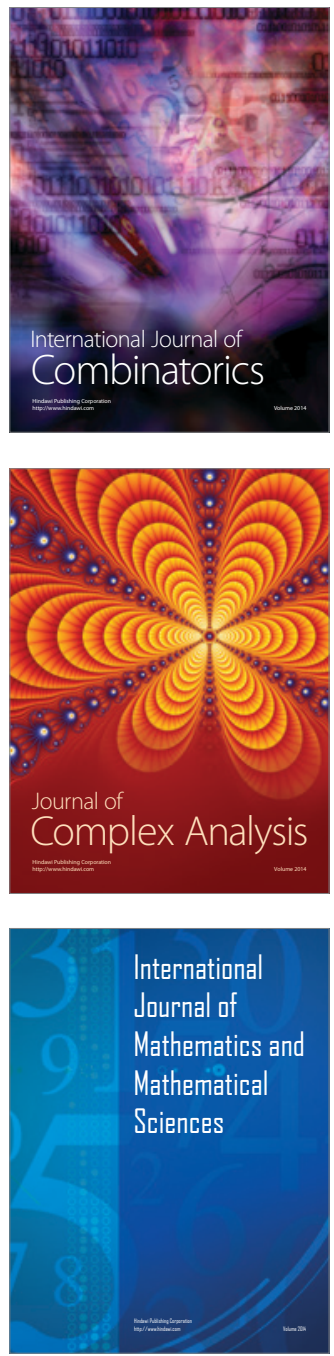
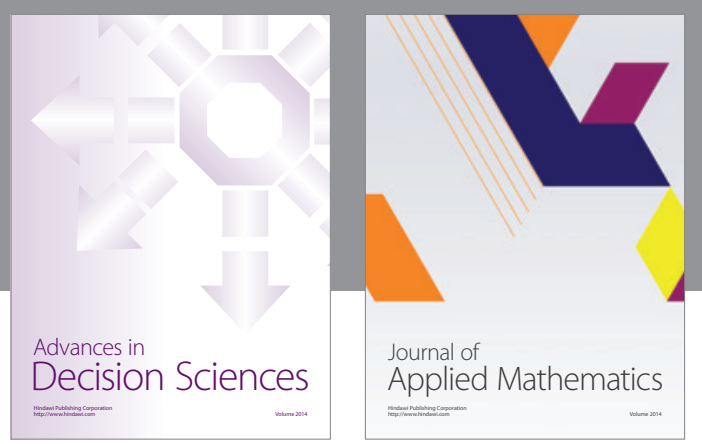

Algebra

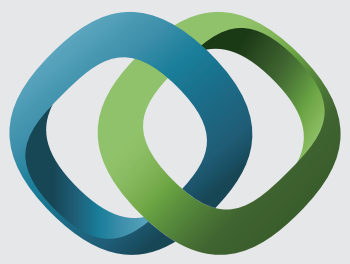

\section{Hindawi}

Submit your manuscripts at

http://www.hindawi.com
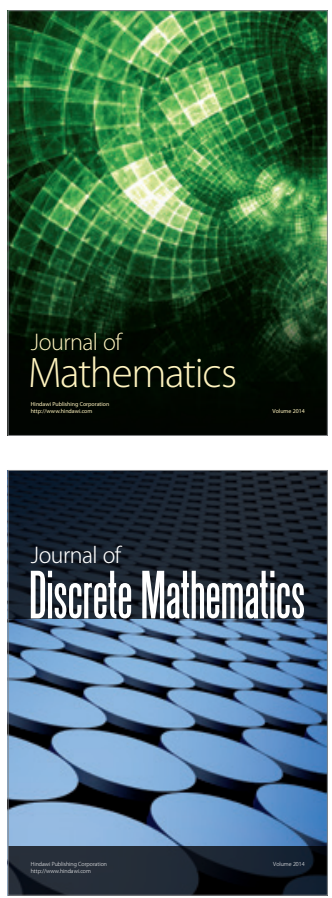

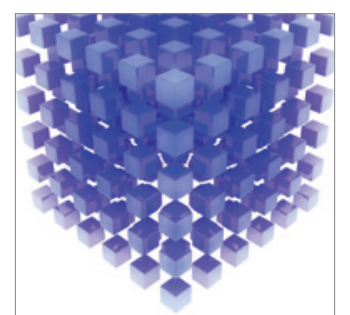

Mathematical Problems in Engineering
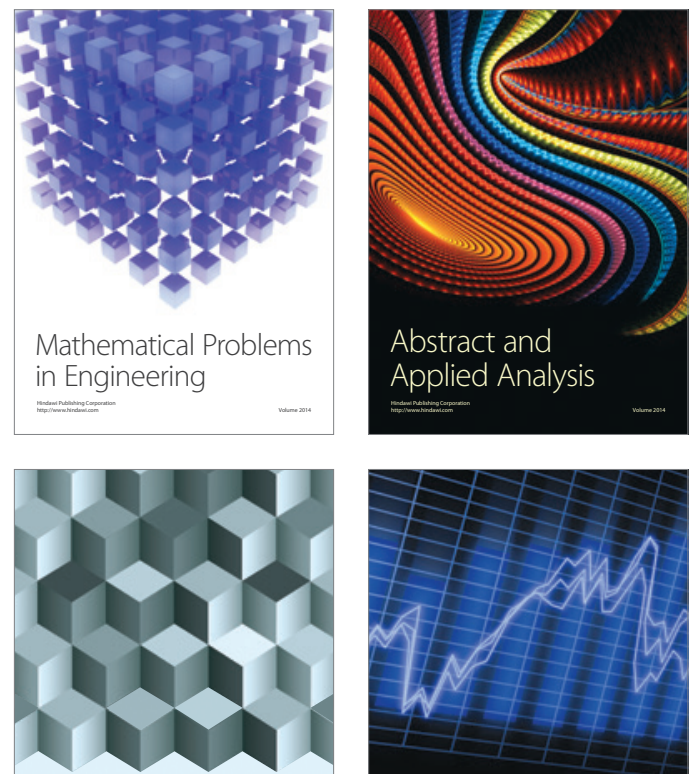

Journal of

Function Spaces

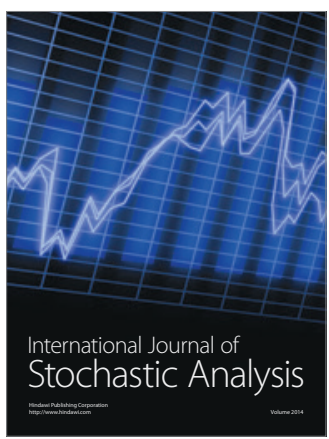

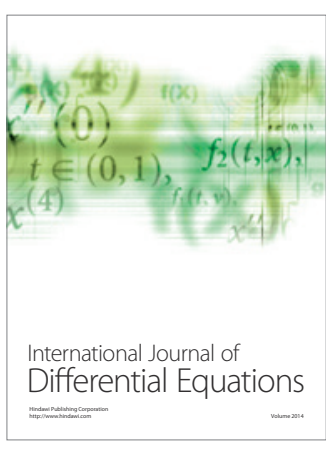
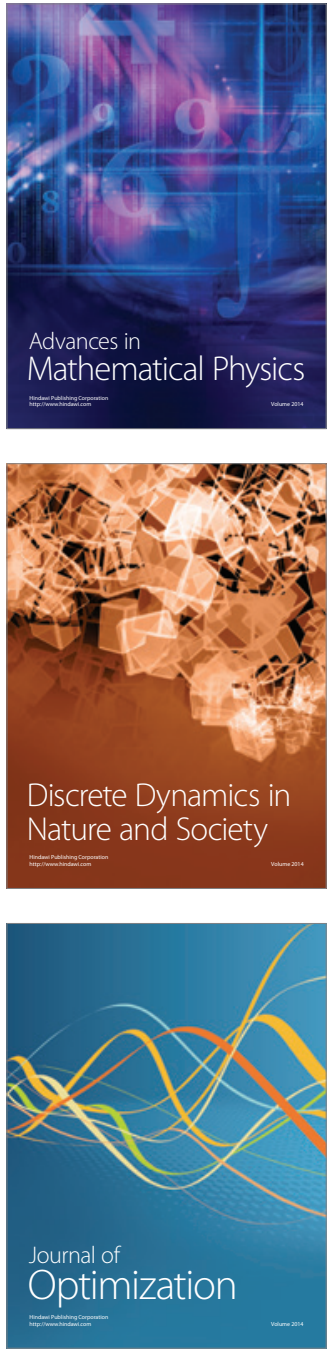